\title{
Heilbehandlung, Heilversuch und Humanexperiment
}

\author{
K. Wasserburg \\ Mainz
}

\section{Zusammenfassung}

Die Thematik dieses Beitrages bezieht sich auf ärztliche Tätigkeitsfelder, die erhebliche juristische Probleme aufwerfen können. Während die Rechtsprechung zur Heilbehandlung kaum noch überschaubar ist, ist es beim Heilversuch oder gar beim Humanexperiment ganz anders: Besonders im letztgenannten Bereich gibt es nur sehr wenig Judikatur, an der sich Ärzte orientieren können. Das Übermaß von Entscheidungen zur Heilbehandlung und ihr weitgehendes Fehlen beim Humanexperiment verunsichert viele Ärzte.

\section{Curative Treatment, Curative Attempt and Human Experiment}

This article deals with a medical field of action that raises essential medical problems. The literature on curative treatment is so vast that it can only rarely be appreciated at a glance, whereas the situation is entirely different in respect of human research and curative attempts. In these two last-named areas only few legal adjudications exist that may guide the medical profession. The flood of literature on curative treatments and the lack of legal decisions on research on humans produce a feeling of insecurity in many physicians.

\section{Einleitung}

Zentrales Rechtsproblem von Heilbehandlung, Heilversuch und Humanexperiment ist insbesondere die schwierige Gewährleistung des Selbstbestimmungsund des Persönlichkeitsrechts des Patienten (Probanden) gegenüber der Absicherung der damit häufig kollidierenden ärztlichen Tätigkeit. Abstrakt läßt sich diese hier notwendige Abwägung in einem Satz formulieren: Die ärztliche Tätigkeit am Menschen ist nur nach wirksam erteilter Einwilligung rechtmäßig (was u. a. vor allem eine ausreichende Aufklärung voraussetzt und außerdem die Beachtung der Sittenwidrigkeitsgrenze erfordert). In den nachfolgenden Ausführungen wird versucht, die Anwendung dieses Satzes auf Heilbehandlung, Heilversuch und Humanexperiment zu skizzieren.

Heilbehandlung ist kaum ohne vorhergehendes medizinisches Experiment denkbar. Die Erfahrungen, die sich aus dem Experiment ergeben, sind Grundlage für den medizinischen Standard, für den Maßstab also, an dem sich jede Heilbehandlung messen lassen muß, an dem sie sich auszurichten hat. Das medizinische Experiment ist Teil der medizinischen Forschung, die ihre Erkenntnisse zunächst also innerhalb vorgegebener Rahmenbedingungen im Experiment anwenden und testen muß, bevor sie sich zum medizinischen Standard entwickeln und so in die Heilbehandlung integriert werden. Medizinische Experimente unterscheiden sich von der Heilbehandlung weniger in der Ungewißheit im Ausgang des Geschehens, auch die übliche Behandlung kann in der Erfolgsprognose unsicher sein. Das Experiment überschreitet jedoch die Grenzen des Standards, indem es sich

Akt. Urol. 26 (1995) 211-215

(C) Georg Thieme Verlag Stuttgart · New York auf empirisch wissenschaftliches Neuland begibt (1). „Das Gegensatzpaar heißt nicht Versuch und Erfolg, sondern Versuchsbehandlung und Standardbehandlung“" (2). Bei den Versuchsbehandlungen wird zwischen Heilversuch und Humanexperiment unterschieden.

Die Integration des Experiments in die Heilbehandlung bedarf der ständigen Kontrolle - der medizinische Standard ist stets an den Erfordernissen der medizinischen Forschung zu messen; auf diese Weise kommt es zur Korrektur, Ergänzung oder Ersetzung medizinisch bereits anerkannter Heilmethoden. Die einmal erfolgte Integration medizinischer Forschungsergebnisse in die Heilbehandlung gewährt also dem medizinischen Standard nicht den Status einer immer unanfechtbaren Erkenntnis - im Gegenteil: Die Forschung mit und am Menschen garantiert den medizinischen Fortschritt - das gilt entsprechend für alle anderen Wissensgebiete. Ihrer fundamentalen Bedeutung gemäß wird die Forschungsfreiheit in Artikel $5 \mathrm{Abs} .3$ GG gerantiert. Einen Gesetzesvorbehalt kennt deses Grundrecht nicht. Die Freiheit der Forschung ist nur durch die damit kollidierenden Grundrechte der Menschen begrenzt, die das Objekt der Forschung sind. Menschenwürde, allgemeines Persönlichkeitsrecht und das Recht auf Wahrung der körperlichen Integrität werden durch Art. 1, 2 GG und zahlreiche einfachgesetzliche Normen (\$ 823 BGB, $§ \S 212$ ff, $223 \mathrm{ff}$ StGB) geschützt (3).

Auf den behandelnden wie den forschenden Arzt können bei Verletzung dieser Rechte, also im Falle eines Körper- oder Gesundheitsschadens des Patienten/Probanden, zivilrechtliche Forderungen sowie strafrechtliche Folgen zukommen: Zivilrechtlich kann sich der Arzt sowohl vertraglichen (z. B. aus einem Behandlungsoder Probandenvertrag) als auch deliktischen Schadensersatzansprüchen (\$823 I, II BGB) ausgesetzt sehen; straf- 
rechtlich kommen Sanktionen wegen eines Körperverletzungs- oder eines Tötungsdeliktes in Betracht ( $\$ 223 \mathrm{ff}$, $212 \mathrm{ff}$ StGB).

Die Begrenzung der Forschungsfreiheit ist absolut notwendig. Dagegen läßt sich nichts einwenden. Das Problem liegt aber in der Art und Weise der Durchführung dieser Begrenzung im Einzelfall. Wie schwierig das ist, zeigt die schon in Kernbereichen keinesweg überzeugende Rechtsprechung (insbesondere bei der Aufklärung und Einwilligung des Patienten sowie der Bewertung der Heilbehandlung als tatbestandsmäßige Körperverletzung), die das Verhältnis zwischen Arzt und Patient (aber auch zwischen Ärzten und Juristen) durchaus belastet. Hinzu kommt, da $B$ es eine gesetzliche Kodifikation von Heilversuch und Humanexperiment nur im Bereich der Arzneimittelforschung ( $\$$ 40, $41 \mathrm{AMG}$ ) gibt. Nur ein Teilbereich der medizinischen Forschungstätigkeit ist also gesetzlich geregelt, darüber hinausgehende Forschung ist es nicht.

\section{Definitionen und Abgrenzungen}

Heilversuch und Humanexperiment sind Grundtypen medizinischer Forschung. Sie sind von der Heilbehandlung abzugrenzen (4). Während die „Heilbehandlung " der Heilung eines individuellen Patienten dient und mit medizinisch anerkannten Mitteln oder Verfahren (Standardbehandlung) durchgeführt wird, hat zwar der „Heilversuch " auch diese Zielsetzung - er dient primär der Heilung des individuellen Patienten; die eingesetzten Mittel oder Verfahren gehören jedoch (noch) nicht zum ärztlichen Standard, der Heilversuch hat also auch einen experimentellen Charakter. Das „Humanexperiment" dient hingegen keinem individuellen Heilungszweck. Es wird zu reinen Forschungszwecken durchgeführt; „nicht mehr der Patient steht zur Behandlung an, sondern ein objektiviertes Individuum, das in den Dienst des medizinischen Fortschritts gestellt wird" (5).

So leicht eine begrifflich-abstrakte Abgrenzung möglich ist, um so schwerer kann die Abgrenzung in der Praxis fallen: Die Abgrenzung ist jedoch von großer Bedeutung, da die Zulässigkeit von Heilbehandlung, Heilversuch und Humanexperiment unterschiedlichen Rechtsregeln unterliegt (6). Dem Arzt muß (müßte) daher jeweils im Idealfall deutlich bewußt sein, unter welche Kategorie sein Handeln fällt. Anderenfalls kann er leicht in die Gefahr eines Rechtsverstoßes geraten. Wesentlich für die Abgrenzung der Heilbehandlung von Versuchsbehandlungen ist auf die Neuheit der angewandten Mittel oder Verfahren abzustellen; damit gewinnt der Begriff des medizinischen Standards Bedeutung. Eine vielzitierte Definition des ärztlichen Standards stammt von Carstensen (7): „Standard in der Medizin repräsentiert den jeweiligen Stand naturwissenschaftlicher Erkenntnis und ärztlicher Erfahrung, der zur Erreichung des ärztlichen Behandlungszieles erforderlich ist und sich in der Erprobung bewährt hat." Untersucht man diese Begriffsbestimmung näher, so kommt man sogleich zu zahlreichen weiteren Problemen, z. B. zu der Frage nach der „Bewährung“ des Erkenntnisstandards (wer legt die „Bewährung“ nach welchen Kriterien fest?) oder zu der Frage, welcher Stand zur Erreichung des Behandlungsziels erforderlich ist. Darauf und auf weitere Punkte kann hier nicht weiter eingegangen werden.
Das bedeutet nicht, daß der Arzt von vornherein auf ein bestimmtes Mittel oder Verfahren festgelegt wäre. Vielmehr ist er in der Auswahl der zur Verfügung stehenden Behandlungsmethoden grundsätzlich frei. „Die Therapiefreiheit findet aber dort ihre Grenze, wo die Überlegenheit eines anderen Verfahrens allgemein anerkannt ist.“ (8).

Man kann abschließend auf die Abgrenzung des BGH zwischen Heilversuch und Humanexperiment verweisen (sogenannte Thorotrast-Entscheidung) (9): Die Abgrenzung erfolgt über die subjektive Zwecksetzung des Arztes - bei einer „konkret-therapeutischen Zwecksetzung“ (Anwendung eines neuen Mittels oder Verfahrens zur Heilung eines individuellen Patienten) liegt ein Heilversuch, bei einer "generell-therapeutischen Zwecksetzung" (die Heilung des Probanden wird nicht angestrebt, die Erprobung dient allenfalls der Heilung künftiger Patienten) ein Humanexperiment vor (10).

\section{Rechtliche Beurteilung von Heilversuch und Humanexperiment}

Zwischen Heilversuch und Humanexperiment muß, wie gesagt, begrifflich unterschieden werden: Das Humanexperiment darf nur unter viel strengeren Voraussetzungen als der Heilversuch durchgeführt werden; darüber hinaus ist sowohl für den Heilversuch als auch für das Humanexperiment danach zu unterscheiden, ob sie innerhalb der Reichweite des AMG liegen oder nicht. Ist das AMG nicht anwendbar, weil es nicht um Arzneimittelprüfung geht, so stellt sich die Frage der entsprechenden Anwendung dieser Regelungen.

\section{Ausgangslage}

Nach der - von der Literatur heftig bekämpften - Rechtsprechung ist die ärztliche Behandlungsmaßnahme, auch eine im Rahmen einer Standardbehandlung, tatbestandlich eine Körperverletzung im Sinne des $\$ \S 223$ ff StGB. Der ärztliche Eingriff bedarf daher einer besonderen Rechtfertigung (11).

In aller Regel wird der Arzt durch die Einwilligung des Patienten in die Behandlung gerechtfertigt. Beruht die Einwilligung auf Willensmängeln (z. B. auf unzureichender Aufklärung über die Behandlung), so ist sie unwirksam, d.h., der Arzt ist bei seinem Eingriff nicht gerechtfertigt (12). Andere Rechtfertigungsgründe wie mutmaßliche Einwilligung oder Notstand kommen nur in Ausnahmefällen in Betracht. Für die mutmaßliche Einwilligung bleibt nur dann Raum, wenn der Patient (z. B. wegen Bewußtlosigkeit) nicht selbst befragt werden kann und sich auch nicht bereits zuvor ausdrücklich gegen eine Behandlung ausgesprochen hat (13).

Außerhalb der üblichen Behandlung ist die Rechtslage noch unübersichtlicher: Fragen der rechtlichen Zulässigkeit der über die dem ärztlichen Standard entsprechende Heilbehandlung hinausgehenden Forschungseingriffe sind nämlich nur im Bereich des Arzneimittelrechts kodifiziert ( $\$ \$ 40,41 \mathrm{AMG}$ ). Im übrigen ist man auf allgemeine Rechtsgrundsätze oder auf Richtlinien ethischer und standesrechtlicher Art angewiesen (14). 
Rechtliche Zulässigkeit von Heilversuch und Humanexperiment nach §\$ 40 f AMG

Das Humanexperiment wird in $\$ 40 \mathrm{AMG}$ geregelt; danach unterliegt es sehr strengen Voraussetzungen. \$41 AMG regelt die Zulässigkeit des Heilversuchs: Hierfür gelten erleichterte Voraussetzungen. Gesetzestechnisch erfolgt die Privilegierung des Heilversuchs in der Weise, daß $\$ 41$ AMG einige (strenge) Regelungen des $\$ 40$ AMG für nicht anwendbar oder nur modifiziert für anwendbar erklärt.

Nur Ärzte mit einer besonderen Qualifikation, nämlich einer mindestens zweijährigen Erfahrung in der klinischen Prüfung von Arzneimitteln (\$ 40I Ziff. 4 AMG) dürfen Heilversuche und Humanexperimente durchführen.

Die Verletzung einzelner dem forschenden Arzt nach den $\$ \$ 40$ f AMG obliegenden Pflichten kann zur Verwirklichung des Straftatbestandes des \$96 Ziff. 10 AMG oder des Ordnungswidrigkeitentatbestandes des \$97 I i. V. m. \$96 Ziff. 10 AMG führen.

\section{Der Heilversuch}

\$ 41 AMG gilt in der klinischen Arzneimittelforschung „,bei einer Person, die an einer Krankheit leidet, zu deren Behebung das zu prüfende Arzneimittel angewendet werden soll“. Es kommt der Grundsatz der therapeutischen Indikation zur Anwendung, d.h., der Arzt darf den Heilversuch nur durchführen, „wenn die Anwendung des zu prüfenden Arzneimittels nach den Erkenntnissen der medizinischen Wissenschaft angezeigt ist, um das Leben des Kranken zu retten, seine Gesundheit wiederherzustellen oder sein Leiden zu erleichtern“ (\$ 41 Ziff. 1 AMG).

Anders als beim Humanexperiment darf der Heilversuch auch bei einer Person durchgeführt werden, die geschäftsunfähig oder in der Geschäftsfähigkeit beschränkt ist ( $\$ 41$ Ziff. 2 AMG). Auch die nach Aufklärung erteilte Einwilligung ist unter erleichterten Voraussetzungen gültig. Es ist auch hier keine Schriftform der Einwilligung erforderlich ( $\$ 41$ Ziff. 6). Unter besonderen Voraussetzungen sind Aufklärung und Einwilligung sogar entbehrlich (§ 41 Ziff. 7 AMG).

An die Aufklärung des Patienten werden beim Heilversuch noch strengere Anforderungen gestellt als bei der Standardbehandlung. Das gilt in um so größerem Maße, je neuer das Medikament ist und je höher die damit verbundenen Risiken sind (15).

\section{Das Humanexperiment}

Das Humanexperiment unterliegt - wie bereits angedeutet - strengeren Voraussetzungen als der Heilversuch: Es ist nur zulässig, wenn die Risiken, die damit für die Person, bei der es durchgeführt werden soll, "gemessen an der voraussichtlichen Bedeutung des Arzneimittels für die Heilkunde ärztlich vertretbar sind“ (\$ 40 I Nr. 1 AMG). Als Probanden kommen grundsätzlich nur voll geschäftsfähige Personen in Betracht; ein Experiment an
Kindern ist nur unter den Voraussetzungen des $\$ 40$ IV AMG erlaubt. Die Probanden dürfen keine auf gerichtliche oder behördliche Anordnung in einer Anstalt Verwahrten sein (\$ 40 I Ziff. 3 AMG).

Das Humanexperiment erfordert grundsätzlich den Abschluß eines Vertrages zwischen Forscher und Proband (16). Der von dem Probanden erteilten Einwilligung muß eine umfassende Aufklärung über „Wesen, Bedeutung und Tragweite" der klinischen Prüfung vorangehen ( $\$ 40$ I Ziff. 2 AMG). Für den Fall, daß bei dem Experiment ein Mensch getötet oder verletzt wird, muß eine Versicherung abgeschlossen werden (\$40 I Ziff. 8, III AMG).

\section{Rechtliche Zulässigkeit von Heilversuchen und Humanexperimenten außerhalb des $A M G$}

Die $\$ \$ 40$ f AMG gelten nur für die klinische Arzneimittelprüfung. Da diese Normen jedoch aus den Grundsätzen des Berufs- und Haftungsrecht der ärztlichen Behandlung entwickelt worden sind, sind sie durchaus verallgemeinerungsfähig (17). „Es gilt, das Recht des medizinischen Neulandschrittes aus der juristischen Dogmatik des Heileingriffes zu entwickeln." (18) Auf dieser Grundlage lassen sich Zulässigkeit und Grenzen von Heilversuch und Humanexperiment außerhalb des AMG bestimmen.

\section{Der Heilversuch}

Der Heilversuch dient dem individuellen Wohl, nämlich der Heilung oder Besserung des Zustandes des Patienten. Daher sind zunächst die Regeln über die Heilbehandlung (Standardbehandlung) anzuwenden (19). Da hier jedoch ein noch nicht erprobtes Medikament oder Verfahren zur Anwendung kommt, sind an die Zulässigkeit des Heilversuchs strengere Anforderungen zu stellen.

Der Heilversuch muß zunächst indiziert, d.h., die Krankheit des Patienten darf mit den Methoden des ärztlichen Standards nicht oder nur noch unzureichend bekämpfbar sein (vgl. \$41 Ziff. 1 AMG). Der verwendeten neuen Behandlung(sart) muß eine wissenschaftlich begründete Prognose eines therapeutischen Nutzens zugrunde liegen (20). Außerdem müssen der zu erwartende Nutzen des Versuchs und dessen Risiko in einem ausgewogenen Verhältnis zueinander stehen; der Heilversuch muß vertretbar sein (21). Auch an die einer wirksamen Einwilligung vorausgehenden Aufklärung werden im Vergleich zur Standardbehandlung erhöhte Anforderungen gestellt: Insbesondere muß der Patient auch über den Versuchscharakter der Behandlung aufgeklärt werden (22).

Ausnahmsweise sind in besonders schweren Fällen Aufklärung und Einwilligung des Patienten entsprechend $\$ 41$ Ziff. 7 AMG entbehrlich, wenn anderenfalls der Behandlungserfolg gefährdet wäre (23).

\section{Das Humanexperiment}

Bei einem Humanexperiment geht es um die generelle Forschung mit und am Menschen. Mit der Heilung eines bestimmten Patienten hat das Experiment 
nichts mehr zu tun. Daher werden an die Zulässigkeit im Vergleich zum Heilversuch noch deutlich höhere Anforderungen gestellt (24). Das entspricht der vom Gesetz in den $\S \S 40,41$ AMG getroffenen Wertung.

Einen wichtigen Anhaltspunkt gibt $\$ 40 \mathrm{I}$ Ziff. 1 AMG, der als Grundgedanke für alle Arten von Humanexperimenten gelten kann (25). Danach ist das Experiment nur zulässig, wenn die von dem Probanden zu tragenden Risiken in einer ausgewogenen Relation $\mathrm{zu}$ dem voraussichtlichen Ertrag stehen, den das Experiment für die medizinische Wissenschaft bringt. Zunächst kommt es also auf eine angemessene Nutzen-Risiko-Relation an (26): Bei der zu treffenden Abwägungsentscheidung hat der Arzt nach Standesrecht das Votum einer Ethik-Kommission einzuholen (27). Die Ethik-Kommission kann dem forschenden Arzt die Verantwortung für seine Entscheidung zwar nicht abnehmen, sie hat jedoch eine wichtige beratende Funktion (28).

Eine Abwägungsentscheidung ist jedoch nicht nur in der Phase vor dem Versuch, sondern auch noch während dessen Verlauf erforderlich: Bestätigt sich im Versuch frühzeitig die Wirksamkeit oder Unwirksamkeit eines Medikamentes oder einer Behandlungsmethode, oder wird das mit dem Versuch für den Probanden verbundene Risiko zu groß, so ist der Versuch abzubrechen. Den forschenden Arzt trifft die Pflicht zur permanenten Verlaufsbeobachtung und Erfolgsbeurteilung (29).

An die dem Experiment vorangehende Aufklärung des Probanden werden sehr große Anforderungen gestellt: Er ist „uneingeschränkt über Art und Bedeutung des Versuchs - einschließlich der möglichen Folgen und Nebenwirkungen - aufzuklären“; es gilt eine „völlig uneingeschränkte Aufklärungspflicht“ (30). Erteilt der so aufgeklärte Proband seine Einwilligung, so ist der forschende Arzt gerechtfertigt, es sei denn, die Sittenwidrigkeitsgrenze des $\$ 226$ a StGB wird nicht beachtet (31).

\section{Aufklärung und notwendige Täuschung}

Die vorangegangenen Ausführungen haben gezeigt, welche große Rolle die auf umfassender Aufklärung beruhende Einwilligung des Patienten oder Probanden in einen Forschungseingriff (bzw. in eine Forschungsbehandlung) hat. Es gibt aber Versuche, die ohne eine reduzierte Aufklärung oder gar Täuschung des Probanden nicht durchführbar sind. Solche Experimente kommen im Rahmen kontrollierter klinischer Versuche vor.

Unter kontrollierten klinischen Versuchen versteht man ,jede bewußte diagnostische oder therapeutische Maßnahme [...], die im Hinblick auf ein bestimmtes Resultat unternommen und auf dieses Ergebnis hin überwacht wird" (32). Die Gewinnung des Resultats und die Überwachung geschieht durch die Bildung zweier Gruppen: an einer Gruppe wird die Maßnahme ausprobiert, eine andere Gruppe (Kontrollgruppe) erhält, sofern vorhanden, eine Standardbehandlung oder ein wirkungsloses Präparat (Plazebo). Von einem Blindversuch wird dann gesprochen, wenn der Patient/Proband nicht weiß, ob er zur Testgruppe oder zur Kontrollgruppe gehört. Um einen Doppelblindversuch handelt es sich, wenn auch der Arzt die Zugehörigkeit der Patienten/Probanden zu den Gruppen nicht kennt (33).

Kontrollierte klinische Versuche werfen verschiedene rechtliche Probleme auf. Am schwerwiegendsten sind die Bedenken hinsichtlich deren Zulässigkeit, wenn dem Patienten zu Forschungszwecken Plazebos verabreicht werden, obwohl eine Standardbehandlung existiert.

Zunächst ist grundsätzlich festzustellen: Versuche, insbesondere der Doppelblindversuch, sind international anerkannt und oftmals die einzig wirksame Methode zur Feststellung der Wirksamkeit neuer Behandlungsmethoden. Die extreme Ansicht von Fincke (34), nach der sich der kontrollierte Versuch in der Regel als strafbares Unrecht erweist, wird von einem Großteil der Literatur zu Recht abgelehnt (35).

Ist ein wirksames Standardpräparat vorhanden, so kann die Durchführung eines Blindversuchs zur Erprobung eines neuen Präparates mittels Plazebo die Körperverletzungstatbestände ( $\$ 223 \mathrm{ff}$ StGB), bei tödlichem Ausgang die Tatbestände der Straftaten gegen das Leben ( $\S 212 \mathrm{ff}$ StGB), verwirklichen. Eine Gesundheitsbeschädigung liegt z. B. vor, wenn durch das Unterlassen einer wirksamen Therapie eine Krankheit gesteigert wird. Umstritten ist, ob hierin eine Körperverletzung durch aktives Tun oder durch Unterlassen in Garantenstellung zu sehen ist (36).

Ist ein Straftatbestand verwirklicht, so kann der forschende Arzt nur durch die Einwilligung des Patienten gerechtfertigt werden. Selbstverständlich ist ein Plazebo-Versuch nicht möglich, wenn der Arzt den Probanden darüber aufklären müßte, daß er nur ein Plazebo erhält. Die Aufklärung muß jedoch den Hinweis enthalten, daß eine Aufteilung der Probanden nach Zufallsgesichtspunkten erfolgt und daß der einzelne Proband somit lediglich der Kontrollgruppe angehört (37).

Hart (38) hingegen scheint das Vorenthalten einer wirksamen Therapie für nicht rechtfertigbar anzusehen. Da es auf diesem Gebiet an höchstrichterlicher Rechtsprechung fehlt, kann von Rechtssicherheit nicht gesprochen werden.

Unproblematischer ist der Einsatz von Plazebos in anderem Zusammenhang: Existiert kein Standardpräparat, so ist die Anwendung eines neuen Präparats in der einen Gruppe und der Einsatz von Plazebos in der anderen durchaus möglich. Das folgt daraus, daß der Patient grundsätzlich keinen Anspruch auf die Behandlung mit noch nicht hinreichend erforschten Behandlungsmethoden und Präparaten hat (39). Schließlich lassen sich bei der Abgabe von Plazebos mittels des „Plazebo-Effektes“ durchaus im Einzelfall echte therapeutische Erfolge erzielen. In diesen Fällen kann der Einsatz von Plazebos sogar medizinisch indiziert sein. (40).

\section{Die Ethik-Kommission}

Nach heutigem Standesrecht hat der forschende Arzt bei ethischen Fragen, die seine Arbeit auf- 
wirft, die Hilfe von Gutachterausschüssen (sog. Ethik-Kommissionen) in Anspruch zu nehmen. Eine Verpflichtung, daß sich der am Menschen Forschende vor der Durchführung klinischer Versuche durch eine Ethik-Kommission beraten lassen muß, hat der Weltärztebund schon in der Revidierten Deklaration von Helsinki 1975 (41) gefordert. Die Verpflichtung wurde vom deutschen Ärztetag 1985 als $\$ 1 \mathrm{IV}$ in die Musterberufsordnung aufgenommen. $\mathrm{Zu}-$ nächst noch als Soll-Vorschrift konzipiert, wurde der $\$ 1$ IV Musterberufsordnung (MBO) 1988 in eine Muß-Bestimmung umgewandelt (42).

Mit der Übernahme der Empfehlung des Weltärztebundes in die Berufsordnung der einzelnen Bundesländer ist die Anrufung der Ethik-Kommission standesrechtliche Pflicht geworden. Bei einer Verletzung der Pflicht drohen standesrechtliche Sanktionen (43). Ferner können sich aber auch arzthaftungsrechtliche Konsequenzen ergeben (44).

\section{Zusammenfassung}

Zusammenfassend ist festzustellen, daß eine Schaffung ausreichender Rechtsgrundlagen Ärzten vordergründig zwar mehr Sicherheit geben könnte, andererseits wären Vorschriften häufig allzu einengend, sie könnten wesentliche Forschungsansätze erheblich beeinträchtigen oder sogar verhindern. Es ist nur schwer vorstellbar, wie gesetzliche Regelungen, die den Schutz des Patienten bzw. Probanden stark berücksichtigen müssen, mit der Forschungs- und Wissenschaftsfreiheit abstrakt, d.h., durch Normsetzung, in befriedigender Weise in Einklang gebracht werden können. Die Frage nach Zulässigkeit und Grenzen ärztlichen Handelns generell - besonders aber beim Humanexperiment - muß im Einzelfall entschieden werden. Die hierauf beruhende rechtliche Unsicherheit muß der Arzt auf sich nehmen. Außerdem spricht gegen eine gesetzliche Regelung beim Heilversuch und Humanexperiment die schief gelaufene Entwicklung des Arztrechts insgesamt (vor allem: Heilbehandlung als Tatbestand der Körperverletzung und die unübersehbare, häufig übertriebene und zudem teilweise auch schwankende Kasuistik zum Recht des Patienten auf Aufklärung und Einwilligung).

\section{Anmerkungen}

${ }^{1}$ Laufs: Arztrecht, Rn. 674

2 Deutsch: Medizin und Forschung vor Gericht, S. 42

3 Pabst: S. 53 ff; Keller: MedR 1991, 11

4 Laufs: Arztrecht, Rn. 675

5 Keller: MedR 1991, 11, 13

6 Hart: MedR 1994, 94

7 DÄBI: 1989, 2431, 2432

8 Laufs: In: Laufs/Uhlenbruck, \$ 3 Rn. 16; vgl. auch Schmid, NJW 1986, 2339; zur Abgrenzung der Versuchsbehandlung von der Außenseiterbehandlung: Hart, MedR 1994, 94, $99 \mathrm{f}$

9 BGHZ 20, 61, 66; vgl. dazu Laufs: Arztrecht, Rn. 675 f m.w. N. über die historische Entwicklung dieser Unterscheidung

10 Schimikowski, S. 9 ff; vgl. ausführlich zu den Begriffen und zu ihrer Abgrenzung: Hart: MedR 1994, 94 ff; Keller: MedR 1991, 11, 13; Laufs: VersR 1978, 385, $387 \mathrm{ff}$; ders., Arztrecht Rn. $675 \mathrm{ff} ;$ Pabst: S. 54; Trockel: NJW 1979, 2329, $2331 \mathrm{f}$

11 Ständige Rechtsprechung seit RG St 25, 375; diese Ansicht wird von großen Teilen der Literatur allerdings mit überzeugenden Gründen angegriffen; vgl. Dreher/Tröndle: $\$ 223$ Rn. 9a, 9 m. w. N; LK-Hirsch, Vor $§ 223$ Rn. 3 ff; Schönke/Schröder-Eser: $\S 223 \mathrm{Rn} .27 \mathrm{ff}$ m. w. N.

12 Schönke/Schröder-Eser: $\$ 223$ Rn. 39

13 Schönke/Schröder-Eser: $\$ 223 \mathrm{Rn} .38$; ausführlich zur Aufklärung und den in Betracht kommenden Rechtfertigungsgründen, Schimikowski: S. $18 \mathrm{ff}$

14 Keller: MedR 91, 11, 14

15 Laufs: Arztrecht, Rn. 679

16 Laufs: Arztrecht, Rn. 680; Hart: MedR 1994, 94, 101

17 Hart: MedR 1994, 95, 95; vgl. auch Kollhosser/Krefft: MedR 1994, S. $94 \mathrm{ff}$.

18 Laufs: Arztrecht, Rn. 689; vgl. ders.: VersR 1978, 385, 388

19 Keller: MedR 91, 11, 14

20 Hart: MedR 1994, 94, 101; Versuche „ins Blaue hinein“ sind also unzulässig

21 Keller: MedR 1991, 11, 15; Laufs: Arztrecht, Rn. 690

22 Hart: MedR 1994, 94, 101

23 Laufs: Arztrecht, Rn. 694; zu dem Wegfall der Aufklärungspflicht unter dem Gesichtspunkt des „therapeutischen Privilegs“: Schönke/Schröder-Eser: \$ 223 Rn. 42

24 Keller: MedR 1991, 11, 15

25 Ähnlich auch Laufs: Arztrecht, Rn. 682; Hart: MedR 1994, 94, 104

26 vgl. Giesen: S. 71

27 Dazu mehr unten

28 Laufs: Arztrecht, Rn. 683 f; Keller: MedR 1991, 11, 15

29 Hart: MedR 1994, 94, 96

30 Giesen: S. 70

31 LK-Hirsch: $\S 226$ a Rn. 47; Schönke/Schröder-Eser: $\$ 223$ Rn. 50; kritisch Schimikowski: aaO S. 44 m.w.N.

32 Deutsch: JZ 1980, 289, 290

33 Deutsch: aa0

34 NJW 1977, 1094

35 Deutsch: JZ 1980, 289, 291; Samson, NJW 1978, 1182, 1183; von einer grundsätzlichen Zulässigkeit kontrollierter Studien gehen z. B. auch Laufs: Arztrecht, Rn. 697 f; Trockel: NJW 1979 , 2329, 2332; Keller: MedR 1991, 11, 16 und Hart: MedR 1994, 94,102 ff aus

36 für aktives Tun: Samson: NJW 1978, 1182, 1184; für Unterlassen: Schimikowski: S. 13

37 Samson: NJW 1978, 1182, 1185 f; Keller: MedR 1991, 11, 16; Deutsch: JZ 1980, 289, 292 f; Trockel: NJW 1979, 2329, 2332; Laufs: Arztrecht, Rn. $697 \mathrm{f}$

38 MedR 1994, 94, 98, Fußnote 48

39 Samson: NJW 1978, 1182,1184 f; Laufs: Arztrecht, Rn. $698 \mathrm{f}$

40 Samson: NJW 1978, 1182, $1186 \mathrm{f}$

41 B-Anzeiger Nr. 152 vom 14. 8. 1976, S. $3 \mathrm{f}$

42 Deutsch: VersR 1989, 429, 430; 1993 hat auch die Landesärztekammer Rheinland-Pfalz den \$1IV ihrer Berufsordnung geändert, so daß er nunmehr folgenden Wortlaut hat: „Der Arzt muß sich vor der Durchführung klinischer Versuche am Menschen oder der epidemiologischen Forschung mit personenbezogenen Daten durch eine bei der Ärztekammer oder bei einer medizinischen Fakultät gebildeten Ethik-Kommission über die mit seinen Vorhaben verbundenen berufsethischen und berufsrechtlichen Fragen beraten lassen." Unter den ständigen Mitgliedern der derzeitigen Ethik-Kommission bei der Landesärztekammer Rheinland-Pfalz befindet sich neben 10 Medizinern auch ein Jurist - Ärzteblatt Rheinland-Pfalz 7/1994, S. 218

43 Deutsch: VersR 1989, 429, 430

44 Für Ansprüche des Patienten/Probanden aus $\$ 823$ I BGB wohl Hart: MedR 1994, 94, 102; für einen Anspruch aus $\$ 823$ II i. V.m. $\$ 1$ IV der Berufsordnungen der Ärztekammer: Taupitz: Standesordnungen, S. $1321 \mathrm{f}$

\section{Dr. K. Wasserburg}

Rechtsanwalt

Adam-Karrillon-Straße 23

55118 Mainz 